\title{
Phase II trial of S-1 for neoadjuvant chemotherapy against scirrhous gastric cancer (JCOG 0002)
}

\author{
Taira Kinoshita ${ }^{1}$, Mitsuru Sasako ${ }^{2}$, Takeshi Sano $^{3}$, Hitoshi Katai $^{4}$, Hiroshi Furukawa $^{5}$, Akira Tsuburaya ${ }^{6}$, \\ Isao Miyashiro ${ }^{7}$, Masahide KajI ${ }^{8}$, and Motoki NinOmiYa ${ }^{9}$ (on behalf of the Gastric Cancer Surgery Study Group \\ of the Japan Clinical Oncology Group) \\ ${ }^{1}$ Department of Surgical Oncology, National Cancer Center Hospital East, 6-5-1 Kashiwanoha, Kashiwa, Chiba 277-8577, Japan \\ ${ }^{2}$ Department of Surgery, Hyogo College of Medicine, Kobe, Japan \\ ${ }^{3}$ Department of Surgery, Cancer Institute Hospital, Tokyo, Japan \\ ${ }^{4}$ Department of Surgical Oncology, National Cancer Center Hospital, Tokyo, Japan \\ ${ }^{5}$ Department of Surgery, Sakai Municipal Hospital, Osaka, Japan \\ ${ }^{6}$ Department of Surgical Oncology, Kanagawa Prefectural Cancer Center Hospital, Yokohama, Japan \\ ${ }^{7}$ Department of Surgery, Osaka Medical Centre for Cancer and Cardiovascular Diseases, Osaka, Japan \\ ${ }^{8}$ Department of Surgery, Toyama Prefectural Central Hospital, Toyama, Japan \\ ${ }^{9}$ Department of Surgery, Hiroshima City Hospital, Hiroshima, Japan
}

\begin{abstract}
Background. The prognosis of scirrhous gastric cancer remains poor despite extended surgery or adjuvant or neoadjuvant chemotherapy. A pilot study of S-1 (TS-1; Taiho Pharmaceutical, Tokyo, Japan), an oral 5-fluorouracil derivative, for neoadjuvant chemotherapy unexpectedly showed good response and a promising effect on survival. Therefore, the Japan Clinical Oncology Group conducted a phase II trial to confirm the efficacy of S-1 for neoadjuvant chemotherapy against resectable scirrhous gastric cancer.

Methods. Patients were eligible if they had typical scirrhous gastric cancer invading more than half of the stomach, and resectable disease confirmed by laparoscopic staging. The treatment schedule consisted of two courses (each, 4-week administration and 2-week withdrawal) of S-1 (100-120 mg/ body per day), followed by radical surgery.

Results. Fifty-five eligible patients were registered. Three completed only one course of the neoadjuvant chemotherapy, whereas 52 completed two courses. Toxicity was acceptable, with a few grade $3(5.5 \%)$ events, but no grade 4 adverse events. The response rate was $32.6 \%$ in 43 evaluable patients. Of the 55 patients, 2 refused operation, 1 developed lung metastasis, and 52 underwent laparotomy. The curative resection rate was $80.8 \%$, with acceptable morbidity and no mortality. The survival curve at 2 years' follow up showed a better survival rate than that of the historical controls, but did not reach the expected survival rate.

Conclusion. S-1 neoadjuvant chemotherapy appeared feasible and showed positive effects against scirrhous gastric cancer; however, the survival rate with S-1 did not reach the expected rate required when selecting an agent for a phase III trial to confirm the effectiveness of neoadjuvant chemotherapy against scirrhous gastric cancer.
\end{abstract}

Offprint requests to: T. Kinoshita

Received: September 16, 2008 / Accepted: November 27, 2008
Key words Scirrhous gastric cancer - Neoadjuvant chemotherapy $\cdot$ S-1

\section{Introduction}

Scirrhous gastric cancer, also known as linitis plastica or Borrmann type 4 , is a special type of stomach cancer known for its very poor prognosis. It is very difficult to identify this cancer in its early stage, and even aggressive surgical procedures and adjuvant chemotherapies have not considerably improved the survival rate in patients with this neoplasia. Owing to its low incidence, only a few drug trials against this neoplasia have been conducted thus far. On the other hand, several studies of neoadjuvant chemotherapy against scirrhous gastric cancer have suggested the efficacy of such treatment [1-4]. However, all these studies involved a small sample size and they usually did not determine the survival benefits of such treatment. Furthermore, a phase II trial of sequential high-dose methotrexate and fluorouracil combined with doxorubicin (FAMTX) for neoadjuvant chemotherapy has shown moderate toxicity and no survival benefits [5]. Interestingly, S-1, which is a dihydropyrimidine dehydrogenase (DPD)-inhibitory fluoropyrimidine, has shown the highest response rate among many oral anticancer agents against unresectable advanced gastric cancer in early and late phase II trials [6-8]. In these late phase II trials, S-1 showed a 33\% response rate against scirrhous gastric cancer. Because of the reported promising effects of S-1 for neoadjuvant chemotherapy against scirrhous gastric cancer in a previous pilot study [9], the Japan Clinical Oncology Group 
(JCOG) decided to conduct a phase II trial to determine survival benefits of S-1 treatment.

\section{Patients, materials and methods}

\section{Patient eligibility}

Patient eligibility required the fulfillment of the following criteria: histologically confirmed gastric adenocarcinoma; potentially resectable laparoscopy-confirmed typical scirrhous gastric cancer (without definitive ulceration) that invaded more than half of the stomach; received no prior treatment; 70 years or younger; Eastern Cooperative Oncology Group performance status of 0 or 1 ; and oral intake possible. Patients also had to have adequate organ functions (creatinine clearance, $\geq 50 \mathrm{ml} / \mathrm{min}$; blood urea creatinine, within the institutional limit; GOT and GPT, within twice the institutional limit; leukocytes, $3500 / \mathrm{mm}^{3} \leq$ leukocyte $<12000 / \mathrm{mm}^{3}$; hemoglobin, $\geq 9.0 \mathrm{~g} / \mathrm{dl}$; thrombocytes, $\geq 1000000 / \mathrm{mm}^{3}$; total bilirubin, within twice the institutional limit; and normal electrocardiogram).

Diagnostic and staging procedures included physical examination, barium gastrography, endoscopy, chest Xray, abdominal computed tomography (CT) scan, and laparoscopy with cytological examination of peritoneal washing of the Douglas pouch. Patients with positive cytology on peritoneal washing and potentially resectable disease without visible peritoneal dissemination were also included in the study.

This study was approved by the Institutional Review Board, and written informed consent was obtained from all patients.

\section{Treatment schedule}

Chemotherapy consisted of two courses (4-week administration and 2-week withdrawal) of S-1 at 100-120 mg/ body per day. After two courses of neoadjuvant chemotherapy, patients were reevaluated for the presence of potentially resectable disease and those who were positive underwent laparotomy. Because two patients underwent endoscopic examination after one course of chemotherapy and stopped chemotherapy due to progressive disease, the treatment protocol was revised such that the evaluation of the effect of neoadjuvant chemotherapy should be carried out only after two courses and only by fluoroscopic examination. If indicated, patients received curative or palliative resection or exploratory laparotomy within 14 days after completing the second course of adjuvant chemotherapy. Patients with curative resection were followed up without any adjuvant chemotherapy every 3 months until cancer relapse.

\section{Evaluation of response and toxicity}

Potentially resectable scirrhous gastric cancer usually shows no measurable lesions, except for primary foci. We decided to evaluate the response of only primary foci following chemotherapy. Because it is very difficult to evaluate the response of the primary foci using the Response Evaluation Criteria in Solid Tumors criteria, we used a National Institutes of Health (NIH) image to calculate the barium-filling area or whole stomach on a double-contrast fluoroscopic examination study, as well as to compare the area before and after chemotherapy. Responses were classified as partial response (PR), more than $50 \%$ increase in the area after chemotherapy; stable disease (SD), 0 to less than $50 \%$ increase in the area; and progressive disease (PD), any decrease in the area and the appearance of new lesions. National Cancer Institute Common Toxicity Criteria ver2.0 were employed for determining chemotherapy toxicity.

Pathological assessment was performed to evaluate disease extent, resection margins, and response to chemotherapy as evidenced by the presence of necrotic and cancer cells. The pathological response to chemotherapy was classified according to the following criteria provided by the Japanese Gastric Cancer Association [10]: grade 0 , absence of necrosis or degeneration; grade $1 \mathrm{a}$, necrosis or degeneration is observed in less than one-third of the tumor; grade $1 \mathrm{~b}$, less than two-thirds and more than one-third of the tumor show necrosis or degeneration; grade 2, more than two-thirds of the tumor shows necrosis or degeneration; grade 3, all tumors show necrosis or degeneration.

\section{Historical controls}

Because we applied laparoscopic staging to exclude patients with visible peritoneal dissemination, it was very difficult to find good historical controls. Laparoscopic staging had gained popularity at the commencement of this trial; however, we had no identical historical controls. The historical controls consisted of 241 patients who had the same lesions as those described in the eligibility criteria for this study, and who had no visible peritoneal dissemination at laparotomy without laparoscopic staging, and had been treated at the participating institution during 1991-1993. Data for the historical controls were as follows: 2-year survival rate, $45 \%$; curative resection rate, $90.3 \%$; 30-day operative mortality rate, $1.2 \%$; and in-hospital mortality rate, $3.5 \%$.

\section{Statistical considerations}

The primary endpoint of this study was the 2-year survival rate. Fifty-five patients were required to be registered on the basis of the expectation that the 2-year survival rate of those receiving this neoadjuvant chemo- 
therapy would be $60 \%(15 \%$ higher than that of the historical controls), allowing $10 \%$ of ineligible patients. Survival time was calculated from the initial date of the initiation of neoadjuvant chemotherapy to the date of death or the last follow-up date. Survival data were analyzed according to the method of Kaplan and Meier and then compared with the data of the historical controls.

\section{Results}

\section{Patient accrual}

From March 14, 2001, to February 4, 2003, 55 patients were enrolled in the study from 15 institutions. The mean age was 56 years (range, $31-70$ years).

\section{Neoadjuvant chemotherapy}

The patients were composed of 26 male and 29 female patients. The scheduled two courses of neoadjuvant chemotherapy were performed in 52 patients. The remaining 3 patients received one course, because 2 of the 3 patients were judged to have PD by endoscopic evaluation after one course before the revision of the protocol, and 1 patient was found to have advanced bile duct carcinoma after one course of chemotherapy. These 3 patients received curative resection after one course of neoadjuvant chemotherapy. There was no chemotherapy-induced grade 4 adverse reaction in the cohort. Only 3 patients developed grade 3 adverse reactions (Table 1).

As mentioned earlier, the effect of adjuvant chemotherapy was evaluated from the change in the barium-

Table 1. Adverse reactions

\begin{tabular}{|c|c|c|c|c|c|c|}
\hline & \multicolumn{4}{|c|}{ Grade } & \multirow{2}{*}{$\begin{array}{c}\% \\
\text { Grade } 4\end{array}$} & \multirow[b]{2}{*}{ Total } \\
\hline & 0 & $1-2$ & 3 & 4 & & \\
\hline T. Bil & 32 & 23 & 0 & 0 & 0 & 55 \\
\hline WBC & 42 & 13 & 0 & 0 & 0 & 55 \\
\hline Neutrophils & 42 & 12 & 1 & 0 & 0 & 55 \\
\hline ALT & 43 & 11 & 2 & 0 & 0 & 55 \\
\hline AST & 45 & 9 & 0 & 0 & 0 & 55 \\
\hline $\mathrm{Hb}$ & 48 & 7 & 0 & 0 & 0 & 55 \\
\hline Nausea/vomiting & 36 & 19 & 0 & 0 & 0 & 55 \\
\hline Pigmentation & 44 & 11 & 0 & 0 & 0 & 55 \\
\hline Anorexia & 45 & 10 & 0 & 0 & 0 & 55 \\
\hline Diarrhea & 45 & 10 & 0 & 0 & 0 & 55 \\
\hline Stomatitis & 45 & 10 & 0 & 0 & 0 & 55 \\
\hline General fatigue & 46 & 9 & 0 & 0 & 0 & 55 \\
\hline
\end{tabular}

Only three patients developed grade 3 adverse reactions, and they recovered by withdrawal of S-1

T. Bil, serum total bilirubin; WBC, white blood cell count; ALT, alanine aminotransferase; AST, aspartate aminotransferase; Hb, hemoglobin filling area before and after the chemotherapy, as calculated from the NIH images. Among the 43 patients whose fluoroscopic films could be evaluated, 14 patients (32.6\%) showed more than 1.5 times enlargement of the stomach (PR); 13 patients showed SD (30.2\%), and 16 patients showed PD $(37.2 \%)$.

\section{Operation}

Among the 55 patients, 3 did not undergo operation, because of the refusal of 2 and because the other patient was found to have pulmonary metastases. Fifty-two patients underwent laparotomy, including the 3 patients who received one course of the neoadjuvant chemotherapy. Among the 52 patients, 6 patients did not undergo resection (5, peritoneal dissemination; 1 , unresectable invasion of the duodenum and pancreatic head). Ten patients underwent palliative resection of the main tumor (2, peritoneal dissemination; 6 , positive cytological examination of abdominal washing; 1 , unresectable tumor with severe invasion to the retroperitoneum; 1 , widespread lymph node metastases). The other 36 patients underwent curative total gastrectomy with various combined organ resections $(25$, spleen; 1 , distal pancreas + spleen; 5 , gallbladder; 2 , left adrenal gland; 2 , transverse colon; 1 , pancreatic head and duodenum). Among the 36 patients, only 1 had D1 lymph node dissection and the remaining 35 had D2 or more lymph node dissection.

The mean operation time for curative resection was $214 \mathrm{~min}$ (range, 130-460 $\mathrm{min}$ ) and that for noncurative resection was $295 \mathrm{~min}$ (range, 150-401 $\mathrm{min}$ ). The mean blood loss for curative resection was $586 \mathrm{ml}$ (range, 30$1815 \mathrm{ml}$ ) and that for noncurative resection was $872 \mathrm{ml}$ (range, 230-2100 ml).

Among the 46 patients who underwent resection, postoperative complications were observed in 11 patients $(23.9 \%)$. Overall, there was no mortality and there were no serious complications. The actual complications were as follows: wound infection, deep vein thrombosis, pancreatic fistula, anastomotic ulcer, pneumonia, pulmonary embolism, sepsis, abdominal abscess, liver function disorder, and mycotic uveitis.

Changes in the T, P, and CY (cytological examination of the abdominal washing) factors before and after neoadjuvant chemotherapy are shown in Tables 2 and 3 . With regard to the $\mathrm{T}$ factor, a response was observed in 14 patients; however, cancer progression was observed in 8 patients. In regard to the $\mathrm{P}$ and $\mathrm{CY}$ factors, a response (PR) was observed in only 2 patients; however, 10 showed progressive disease (PD). The other 40 patients showed stable disease (SD).

The pathological therapeutic effects of neoadjuvant chemotherapy were evaluated according the grading described by the Japanese classification of gastric carci- 
noma [10] general rules for gastric cancer study: grade 0,12 patients $(26.1 \%)$; grade $1 \mathrm{a}, 19$ patients $(41.3 \%)$; grade $1 \mathrm{~b}, 4$ patients $(8.7 \%)$, and grade 2,11 patients $(23.9 \%)$.

At the time of the scheduled analyses (March 2005), 10 patients were still alive without recurrence, 13 were alive with recurrence, and 32 had already passed away. The modes of recurrence were as follows: peritoneal, 17 patients; retroperitoneal, 2 patients; local, 1 patient; lymph node, 1 patient.

Table 2. Changes in $\mathrm{T}$ factors before and after chemotherapy

\begin{tabular}{|c|c|c|}
\hline Laparoscopic T & & Pathological T \\
\hline $\mathrm{T} 2: 7$ & & $\mathrm{~T} 2: 11$ \\
\hline $\mathrm{T} 3: 39$ & Chemotherapy & $\mathrm{T} 3: 37$ \\
\hline $\mathrm{T} 4: 5$ & & $\mathrm{~T} 4: 4$ \\
\hline $\mathrm{Tx}: 1$ & & \\
\hline
\end{tabular}

Progression, 8 patients; downstage, 14 patients

Tx, T unknown

Table 3. Changes in $\mathrm{P}$ and $\mathrm{CY}$ factors before and after chemotherapy

\begin{tabular}{lr}
\hline No change or progression (SD and PD) & \\
P0, CY0 $\rightarrow$ P0,CY0 & $37(\mathrm{SD})$ \\
$\mathrm{P} 0, \mathrm{CY} 0 \rightarrow \mathrm{P} 0, \mathrm{CY} 1$ & $2(\mathrm{PD})$ \\
$\mathrm{P} 0, \mathrm{CY} 1 \rightarrow \mathrm{P} 0, \mathrm{CY} 1$ & $3(\mathrm{SD})$ \\
$\mathrm{P} 0, \mathrm{CY} 0 \rightarrow \mathrm{P} 1$ & $4(\mathrm{PD})$ \\
$\mathrm{P} 0, \mathrm{CY} 1 \rightarrow \mathrm{P} 1$ & $4(\mathrm{PD})$ \\
Downstage $(\mathrm{PR})$ & $2(\mathrm{PR})$ \\
$\mathrm{P} 0, \mathrm{CY} 1 \rightarrow \mathrm{P} 0, \mathrm{CY} 0$ & $2(\mathrm{P})$ \\
\hline
\end{tabular}

The survival curves of all patients $(n=55)$ and the historical controls are shown in Fig. 1. The survival curve of the study arm was better than that of the historical controls; however, the survival rate did not reach the expected rate (2-year survival rate: $59 \%$ vs $60 \%$ ).

With regard to the secondary endpoints, the response rate to the neoadjuvant chemotherapy was $32.6 \%$. The rate of postoperative complications was $23.9 \%$, as against $25.7 \%$ in the historical controls. The in-hospital mortality rate was $0 \%$ as against $3.5 \%$ in the historical controls. The curative resection rate was $80.8 \%$, as against $90.3 \%$ in the historical controls.

\section{Discussion}

Despite recent advances in chemotherapy and extended surgery, the treatment outcomes of scirrhous gastric cancer, also known as diffuse gastric cancer, linitis plastica, or Borrmann type 4 in the West, have remained very poor because of the aggressive biological behavior of this tumor. Because of failure to improve survival even with aggressive postoperative chemotherapy, neoadjuvant chemotherapy has been applied to patients with resectable or unresectable scirrhous gastric cancer.

To date, the efficacy of neoadjuvant chemotherapy against scirrhous gastric cancer remains to be established because of the lack of well-validated phase II and phase III studies. The first phase II neoadjuvant chemotherapy trial was reported by Takahashi et al., using FAMTX [5]. In their trial, neoadjuvant chemotherapy was shown to be seemingly feasible against scirrhous
Survival curves $\quad \mathrm{p}=0.245$

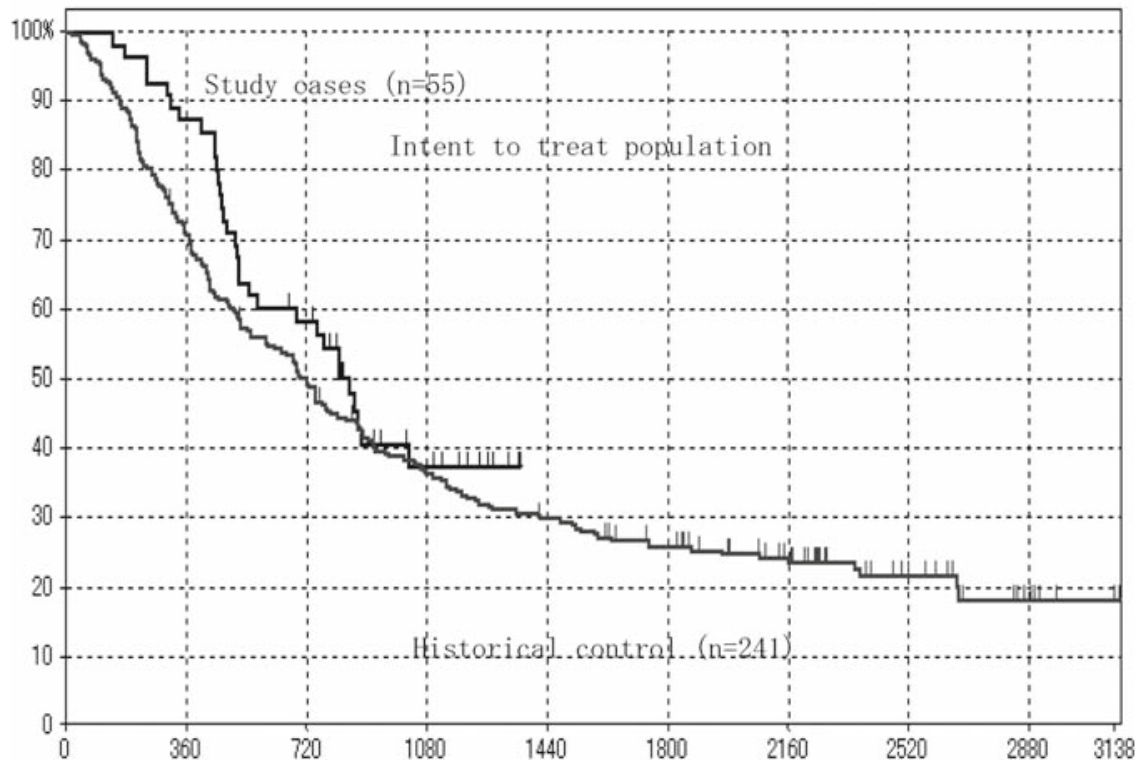

Fig. 1. Survival curves of all patients $(n=55)$ and the historical controls $(n=241)$ 
gastric cancer, producing a higher resectability rate without any increase in morbidity rate. However, an interim analysis of the 2-year survival rate in 20 patients enrolled in the trial showed no improvement over the survival rate of the historical controls. Myelosuppression was the major cytotoxic effect of the FAMTX regimen, and grade 3 or 4 neutropenia was observed in 14 out of the 20 patients $(70 \%)$. Eleven of these 14 patients required granulocyte colonystimulating factor support. The overall response rate was $15 \%$ ( 3 PRs in 20 patients). Eighteen resected specimens showed only marginal histological effects (grades 0 -Ib). For these reasons, Takahashi and co-workers discontinued the trial.

Because S-1 showed promising effects when used for neoadjuvant chemotherapy against scirrhous gastric cancer in a pilot study [9], we decided to conduct a phase II trial of S-1 to determine its beneficial effects on survival. Because of the difficulty in excluding patients with peritoneal dissemination by conventional diagnostic imaging procedures such as CT scan and the use of barium enema, we performed laparoscopic examination to identify and exclude patients with peritoneal dissemination.

At the time of starting the phase II trial, laparoscopic examination for cancer staging was still not a common procedure. Thus, we need to standardize this technique using a video for the quality control of the procedure. Regarding the historical controls, it was not possible to submit patients without peritoneal dissemination to laparoscopic examination, for the same reason. Data for previous patients with the same eligibility criteria and without peritoneal dissemination, confirmed by laparotomy, were collected from the participating institutions. Thus, in the present study, the control group was not identical to the study group.

Neoadjuvant chemotherapy using S- 1 was safe and feasible when compared with other toxic combination chemotherapies. Only a few grade 3 and no grade 4 adverse reactions resulting from cytotoxicity were observed, and no specific morbidity and no increases in morbidity and mortality rates were seen when compared with the data in the historical controls.

Patients with positive cytological examination results were included in this phase II trial. This is the reason why we expected the S-1 neoadjuvant chemotherapy to produce negative cytological examination results. However, the results of the trial, in terms of cytological findings, were not very promising. Without considering the cytological examination results, it can be observed that although there was no significant difference in the curative resection rate between the study group and the historical control group, the curative resection rate in the study group was lower than the expected rate.
From the viewpoint of the pathological therapeutic effects of chemotherapy, S-1 neoadjuvant chemotherapy showed a much better therapeutic effect than FAMTX.

The survival rate of our study group showed a better curve than that of the historical controls; however, it did not reach the expected rate $(P=0.245)$. On the other hand, combination chemotherapy using S-1 and cisplatin (CDDP) showed a markedly high response rate $(76 \%)$ in a phase II trial. Therefore, this combination can be considered more promising than S-1 monotherapy for neoadjuvant chemotherapy against scirrhous gastric cancer. The JCOG has also completed the accrual of patients evaluated in the phase II trial of neoadjuvant chemotherapy using the above S- 1 and CDDP regimen for resectable scirrhous and more-than-8-cm giant type 3 gastric cancer. Because of the superiority of this regimen over S-1 monotherapy in terms of the response rate and pathological therapeutic effects, the JCOG group has already started a phase III trial to confirm the effectiveness of neoadjuvant chemotherapy using S-1 + CDDP as against extended surgery in patients with scirrhous or large type 3 gastric cancer.

In summary, neoadjuvant chemotherapy using S-1 against potentially resectable scirrhous gastric cancer appears feasible and effective; however, in the present phase II trial, the survival rate of the patients did not reach the expected rate. On the other hand, an S-1 + CDDP regimen is now being tested in a phase III trial by the JCOG group as a more promising neoadjuvant regimen.

Acknowledgments This study was supported by a Grantin-Aid for Cancer Research and the Second Term Comprehensive Strategy for Cancer, both by the Ministry of Health, Labour and Welfare, Japan.

\section{References}

1. Mai M, Ogino T, Ueda H, Ooi A, Takahashi Y, Sawaguchi K, et al. Study on neoadjuvant chemotherapy of Borrmann 4 type carcinoma of the stomach and its clinical significance. Nippon Gan Chiryo Gakkai Shi (J Jpn Soc Cancer Ther) 1990;25:586-97.

2. Maeda O, Iwase H, Mamiya N, Nakamura M, Mizuno T, Nishio $\mathrm{Y}$, et al. A case of scirrhous cancer of the stomach which survived for more than 5 years after neoadjuvant chemotherapy with UFT (uracil and tegafur) and cisplatin. Intern Med 2000;9:239-44.

3. Eriguchi M, Osada I, Fujii Y, Takeda Y, Yoshizaki I, Akiyama $\mathrm{N}$, et al. Pilot study for preoperative administration of 1-OHP to patients with advanced scirrhous type gastric cancer. Biomed Pharmacother 1997;51:217-22.

4. Suga S, Iwase $H$, Shimada M, Nishio $Y$, Ichihara $T$, Ichihara $S$, et al. Neoadjuvant chemotherapy in scirrhous cancer of the stomach using uracil, tegafur and cisplatin. Intern Med 1996;35:930-6.

5. Takahashi S, Kinoshita T, Konishi M, Nakagohri T, Inoue K, Ono $\mathrm{M}$, et al. Phase II study of sequential high-dose methotrexate and fluorouracil combined with doxorubicin as a neoadjuvant chemo- 
therapy for scirrhous gastric cancer. Gastric Cancer 2001;4; 192-7.

6. Sugimachi K, Maehara Y, Horikoshi N, Shimada Y, Sakata Y, Miyachi Y et al. An early phase II study of oral S-1, a newly developed 5-fluorouracil derivation for advanced and recurrent gastrointestinal cancers. Oncology 1999;57:202-10.

7. Sakata Y, Ohtsu A, Horikoshi N, Sugimachi K, Mitachi Y, Taguchi T. Late phase II study of novel oral fluoropyrimidine anticancer drug S-1 (1 M tegafur-04 M gimestat- $1 \mathrm{M}$ otastat potassium) in advanced gastric cancer patients. Eur J Cancer 1998; 34:1715-20.
8. Koizumi W, Kurihara M, Nakano S, Hasegawa K. Phase II study of S-1, a novel oral derivative of 5-fluorouracil, in advanced gastric cancer. Oncology 2000;58:191-7.

9. Kinoshita T, Konishi M, Nakagohri T, Inoue K, Oda T, Takahashi S, et al. Neoadjuvant chemotherapy with S-1 for scirrhous gastric cancer. A pilot study. Gastric Cancer 2003;6:40-4.

10. Japanese Gastric Cancer Association. Japanese classification of gastric carcinoma-second English edition-. Gastric Cancer 1998;1:10-24. 